\title{
Local Flow Sensing on Helical Microrobot for Semi-Automatic Motion Adaptation
}

\author{
Antoine Barbot, Dominique Decanini, Gilgueng Hwang
}

\begin{abstract}
Helical microrobots with dimensions below $100 \mu \mathrm{m}$ could serve many applications for manipulation and sensing in small, closed environments such as blood vessels or inside microfluidic chips. However, environmental conditions such as surface stiction from the channel wall or local flow can quickly result in the loss of control of the microrobot, especially for untrained users. Therefore, to automatically adapt to changing conditions, we propose an algorithm that switches between a surface-based motion of the microrobot and a 3D swimming motion depending on the local flow value. Indeed swimming is better to avoid obstacles and difficult surface stiction area but it is more sensitive to the flow than surface motion as rolling or spintop motion. Firstly, we prove the flow sensing ability of helical microrobots based on the difference between the tracked and theoretical speed. For this, a $50 \mu \mathrm{m}$ long and $5 \mu \mathrm{m}$ diameter helical microrobot measures the flow profile shape in two different microchannels. These measurements are then compared with simulation results. Then, we demonstrate both swimming and surface-based motion using closed-loop control. Finally, we test our algorithm by following a 2D path using closed-loop control, and adapting the type of motion depending on the flow speed measured by the microrobot. Such results could enable simple high-level control which could expand the development of microrobots toward applications in complex microfluidic environment.
\end{abstract}

\section{Introduction}

Many potential applications for untethered microrobot moving in liquid have been proposed. They mainly concern biological and medical science to carry out procedure, such as drug delivery (Mhanna et al. 2014; Medina-Sánchez et al. 2016), cargo transport,(Tottori et al. 2012; Zhang et al. 2010a), biopsy (Leong et al. 2009) or gene transmission (Qiu et al. 2015). However, there are only a few examples in which untethered microrobots were successfully used beyond the proof of concept and were directly applied as a tool. For now, the applications are restrained to delicate handling of small crystals (Tung et al. 2014) and stress force studies on microorganisms (Kawahara et al. 2013). This is partly due to the difficulty in producing microrobots that can be moved and controlled in an in-vivo environment without being lost in the flow or becoming permanently stuck to a solid surface. As in-vitro condition simplifies the environment, the integration of microrobots into a microfluidic chip to serve as untethered tools has been investigated. Moreover this platform can propose a common environment for microrobot and biological specimens such as cell (Hagiwara et al. 2011), tissue, or microorganisms (Kawahara et al. 2013).

However, to allow a large range of possible microfluidic chip tasks or even potential in-vivo task, the optimization cannot only come from the environment but also from the microrobot design and control method. In our previous work (Barbot et al. 2016), we show that helical microrobots with different kind of motions have increased robustness and can perform more complicated task. Indeed, the proposed microrobots could choose between propelling itself in 3dimensions (3D) in the liquid (swimming motion) or to propel due to the surface friction with the channel wall in 2D. The different advantages of these two types of motions was essential to succeed in the selective integration (i.e. integration of a single specific microrobot) inside microfluidic chips. In this paper, we propose a semiautomatic control scheme, in which an algorithm chooses instead of the user, the most pertinent motion needed to move along a defined path. The goal is to guarantee that the microrobot cannot be lost due to excessive flow.

For the two motions of our ferromagnetic helical microrobot, the rotation of the magnetic field creates the propelling force and the normal axis of the rotation plane sets the microrobots direction. In our design, a rotating frequency above $50 \mathrm{~Hz}$ guarantees that the microrobot aligns perpendicularly along its primary axis with the magnetic field (Barbot et al. 2017). Therefore, when the magnetic field rotates, the microrobot rotates around its axis plane and its direction is perpendicular to the rotating magnetic field plane. A corkscrew design transforms the rotation into a propelling force and allows the microrobot to swim in 3D. It has been demonstrated with different fabrication methods such as self-scrolling (Zhang et al. 2010b), template electrosynthesis ( $\mathrm{Li}$ et al. 2013) or manual assembly for millimetric scale version (Honda et al. 1996; Mahoney et al.

\footnotetext{
${ }^{1}$ Sunrise Setting Ltd, UK

${ }^{2}$ SAGE Publications Ltd, UK
}

\section{Corresponding author:}

Gilgueng Hwang, Centre for Nanoscience and Nanotechnology, CNRS, Univ. Paris-Sud, Univ. Paris-Saclay, 91460 Marcoussis, France

Email: gilgueng.hwang@c2n.upsaclay.fr 


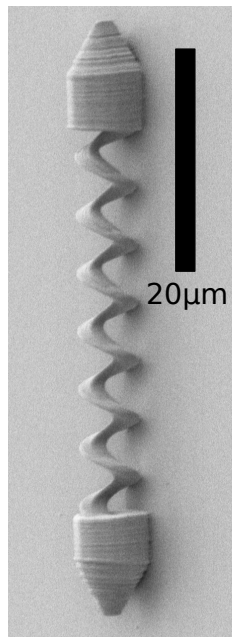

a)

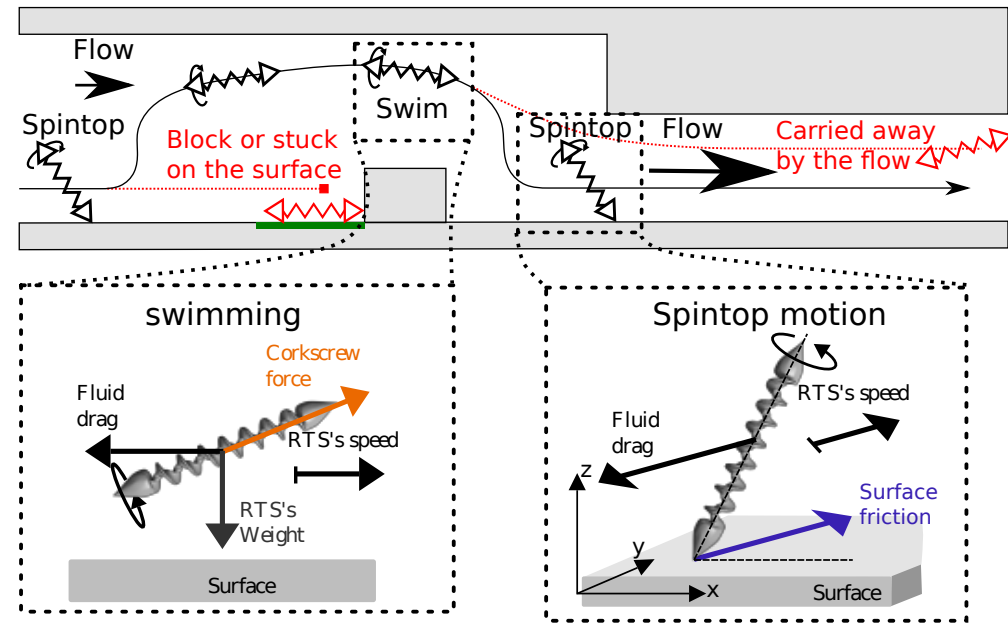

b)

Figure 1. a) SEM picture of the RTS. b) illustration on how the right combination of spintop motion and swim motion can guide the RTS through a complex environment.

2011). Swimming is the first motion that we choose for our control algorithm.

The second motion relies on surface friction to be less impacted by the fluid flow. Rolling, whereby the microrobot touches the surface with its full length, is too hard to control at lower speeds due to the wobbling phenomena. Therefore, for a simpler control we use the "spintop motion". In this motion, the propelling force pushes the microrobot to the surface of the channel so the contact is made only with one extremity of the microrobot. The angle difference with a perpendicular contact between the microrobot and the surface is then used to control the speed at a constant frequency thus avoiding any wobbling issues. More detail on this motion can be found in our previous work (Barbot et al. 2016). It was also reported as a method to move microspheres (Ye et al. 2012). The small contact area between the surface and the microrobot that occurs as a result of this motion is also interesting for biomedical applications and has been used for drilling tissue (Xi et al. 2013) and drug delivery (Srivastava et al. 2016) with magnetic actuated microrobots.

Figure 1 b) shows schematics of these two motions and illustrates their advantages and drawbacks. Swimming allows movements in 3D whereas spintop motion can be blocked by an impassable obstacle or can make the microrobot permanently stuck to the surface. However, the spintop motion is less sensitive to the external flow speed and prevents loss of the microrobot in the stream if the flow becomes too strong. Therefore, we propose to use the microrobot to measure the local flow speed. Then, depending on this speed measurement, the choice between swimming or spintop motion can be automatically made.

Because it can switch from entirely surface friction propel motion (rolling) to entirely fluid motion (swimming) and still offer intermediate motion (spintop motion), this microrobot was called Roll-to-Swim (RTS) (Barbot et al. 2016). We also refer to the refereed article for the magnetic actuation setup and software control. A Scanning Electronic Microscope (SEM) image of our microrobot is presented in Figure 1 a). We can note that the helix is left-handed. Therefore, in swimming the RTS advances forward when the rotation is anticlockwise. For spintop motion, the direction is mainly set by the friction between one head and the surface. So the direction axis makes approximately a -90 degrees angle with the projection of the RTS axis on the surface.

This paper first gives a brief presentation of the fabrication methods of the RTS and the microfluidic chip as well as the steps to integrate one RTS inside the chip. Then, spintop motion and swimming motions are presented as well as their associated closed-loop control system. The swimming closed-loop controller is described in more in detail as it offers a new approach by compensating the gravity in openloop using a prior characterization of the helical microrobot. Then, this closed-loop control is used to demonstrate the ability of the RTS to measure local flows by retrieving different flow profiles in $1 \mathrm{~mm}$ high channels.

Finally, this new ability of local flow sensing in swimming motion is used to propose and test a new control algorithm that avoids losing the RTS when the flow becomes too large. The key part of this algorithm is to detect excessive flow conditions and to trigger a transition to continue the desired path along the microchannel surface using spintop motion. The automatization to come back from spintop motion to a swimming closed loop path following is also demonstrated. However, the choice to trigger this transition is for now manual. Indeed the automatization of this task which beneficial to the microrobot robustness requires deeper study of the surface mechanism. This is out of the scope of this paper. Therefore, The objective of this article is to demonstrate that the robustness of the swimming motion under flux is increased by the flow sensing capability. The obstacle detection and avoidance are not the goal of this paper since the principle targeted motion is 3D swimming which can avoid unknown surface stiction. To limit the probability of surface stiction, the spintop motion is preferred to the rolling motion as the contact with the surface is much smaller. However, for large flux, the rolling motion could be 
an interesting solution as it is even less impacted by the flow than the spintop motion.

\section{System and Control}

\section{Microrobot and Chip Fabrication}

Our helical microrobot (RTS) is fabricated with twophoton lithography using a commercially available system (nanoscribe: photonic professional). Figure $2 \mathrm{~A}$ presents the different steps of this fabrication. First a photo sensitive resist (IPG 780) is deposited on a glass substrate. Then the laser is focused on a precise position to polymerize an elementary volume of resist; this volume is referred as the voxel. By moving the laser, the shape of the helical microrobot can be achieved. Finally, a development step removes the uncured resist. In order to be able to move the microrobot with a magnetic field a Physical Vapor Deposition (PVD) process deposits a ferromagnetic layer on the microrobot. Firstly, $20 \mathrm{~nm}$ of chrome is deposited to ensure a good adhesion both at $0^{\circ}$ and $75^{\circ}$ inclinations, then $100 \mathrm{~nm}$ of nickel is deposited at $0^{\circ}$ and $75^{\circ}$ to form the ferromagnetic layer. This design method of helical magnetic microrobot was firstly reported by Totorri et al.(Tottori et al. 2012) The microfluidic chip is designed with a soft polymer material called polydimethylsiloxane (PDMS). We choose this material for the simplicity of it curing which allows for simple mass production using a master mold. Moreover the transparency of this material is compatible with optic feedback and its elasticity and low Young"s modulus allow for simple valve systems by simply compressing microchannels.

Figure 2 B shows the different fabrication steps of the microfluidic chip. First, the master mold is made with a micromilling machine. Then uncured PDMS mixed with a curing agent is poured on this mold and put in a $75^{\circ} \mathrm{C}$ oven to cure for one hour. After this, the PDMS is removed from the mold and openings are performed with a cutting tool to allow fluid injections and an open chamber for the integration of the microrobot. Finally, the chip is closed by bounding the PDMS to a glass substrate with an oxygen plasma treatment.

Figure $2 \mathrm{C}$ sketches the integration of one RTS inside the microfluidic chip. For this integration, the chip is placed on a magnetic setup made with an assembly of three orthogonal pairs of Helmholtz coils. Therefore, this setup can produce a homogenous magnetic field in any direction. To move and control the RTS, we command this setup to produce a rotating magnetic field. Indeed, as the RTS is ferromagnetic it remains aligned with the field and also rotates at the same frequency. This rotation results in the displacement of the RTS, either due to the friction with the surface which results in rolling or spintop motion, or by the interaction of the helical shape with the fluid that creates a propelling force and make the RTS swim in 3D in the fluid. By combining these different motions the mobility of the RTS is increased and it can reach any place inside the microfluidic chip. For this, a tip first detaches one RTS from the fabrication surface placed on an open chamber of the chip. Then, this RTS swims until the entrance of a microchannel connecting the open chamber to the isolated part of the chip. At this point the RTS can move using the friction surface to easily overcome the residual parasite flow on the microchannel. When the RTS is inside the microfluidic chip the connection with the open chamber is closed by compressing the connecting microchannel with a screw. For more details on the fabrication protocol, the control, and integration of the microrobot inside the microfluidic chip we refer to our previous work (Barbot et al. 2016). This work also explains in detail the different possible motions of the RTS with their different advantages and drawbacks.

\section{Motion Introduction and Closed-loop control}

The goal of this paper is to propose spintop motion as an automatic path following alternative to swimming in strong flow. Therefore, to propose an experimental demonstration we must first develop a closed-loop control for both motions. In this subsection we also want to briefly stress the differences between swimming and spintop motion.

Spintop motion is a friction based motion where only the tip of the microrobot touches the surface. The rotation of the RTS creates a friction force at this contact point which propels it along the surface. As a spintop toy, the RTS moves only if the angle between the surface and its axis is not perpendicular. In other words, if the surface is horizontal and the RTS stands vertically while rotating, it will not move.

A fine model of the spintop motion capable of estimating a quantitative behavior of the RTS is for now impossible. Indeed this motion is a non-linear combination of the microrobot surface friction and the viscous propelling force. The non-linearity is due to the impact of the surface on the flow distribution as well as to the propelling force which increase the friction by pushing the RTS toward the surface. In Appendix B.1, we also report a series of experiments demonstrating that two friction regimes can exist: One which is purely based on viscous friction and another one with a contact friction well modeled by a Coulomb law.

Therefore, we based our control on an empirical model of the spintop motion. In the vicinity of the horizontal (in practice around 20 degrees), we noted that the speed evolves linearly with the change in the pitch angle of $\theta$ (Barbot et al. 2016) Therefore, we use this region to control the speed of the RTS. Firstly, the position error between the real position and the command position $\varepsilon_{x}$ and $\varepsilon_{y}$ are sent to two different Proportional Integrative Derivative (PID) correctors with the same tuning. From these PID, we obtain respectively $C_{x}$ and $C_{y}$ which correspond to the two components of a vector, the angle of which set the control direction and the length set the control speed. Therefore, the following control formula for the pitch $(\theta)$ controlling the speed arises:

$$
\theta=\frac{\pi}{2}-\sqrt{C_{x}^{2}+C_{y}^{2}}
$$

A cap is set on $\theta$ value to force the value 0 in case Equation 1 give a negative result for large $C$ value.

Secondly, the yaw angle (illustrated by $\psi$ in Figures 3 a) ) is used to set the moving direction of the RTS on the surface. Between this angle which is set by the normal of the rotating magnetic field plane and the RTS direction, there is an angle slightly inferior to -90 degrees ( It would be superior to 90 degrees for a right hand helical microrobot). This is the result of the summation of the rotating component of the RTS head on the surface that provides a rolling like motion and of the propelling force of the helix. 

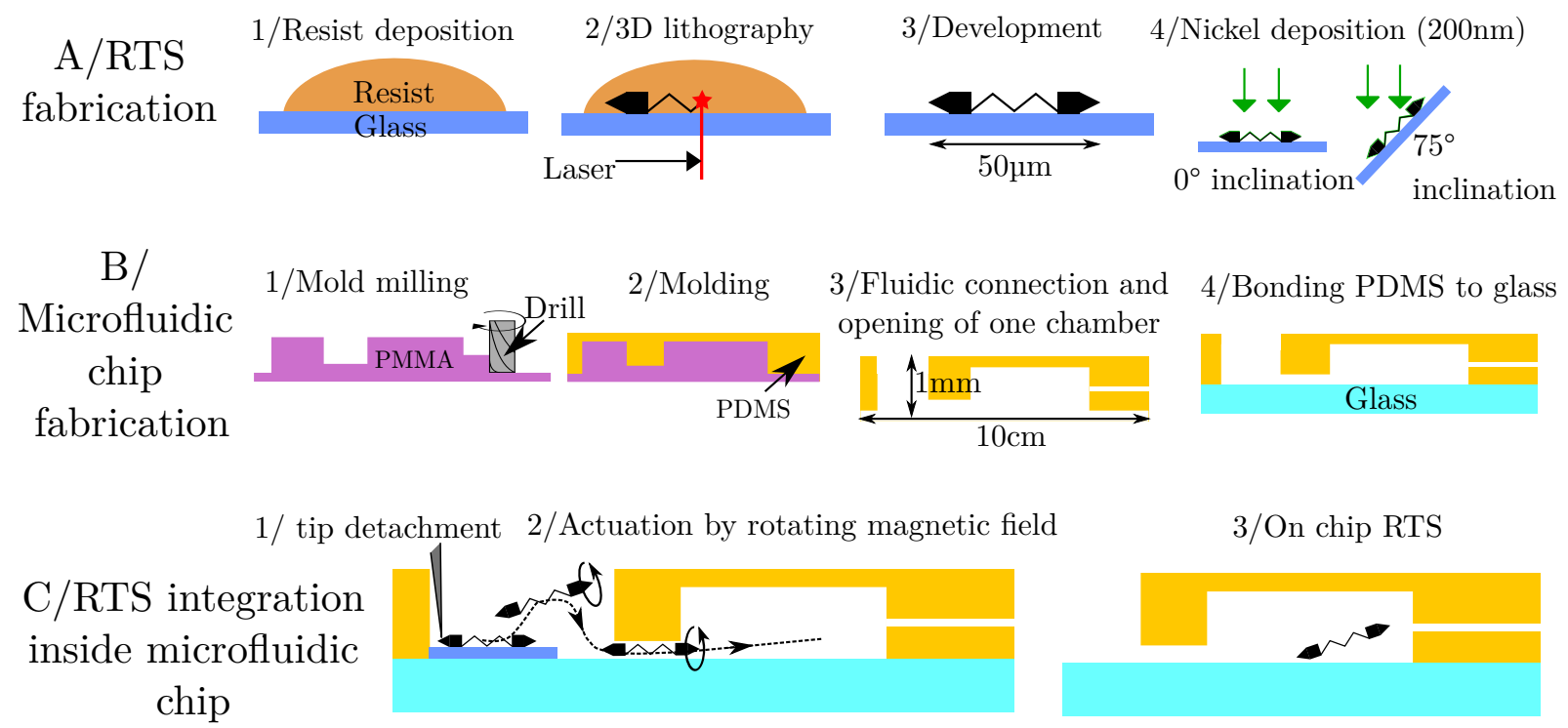

Figure 2. Fabrication steps to obtain a helical microrobot embedded in a microfluidic chip. I) presents the steps link to the helical microrobot fabrication, II) presents the microfluidic chip fabrication steps, III) present the integration of the microrobot inside the chip.

To compensate for this effect, an offset is added on the control $\psi$. Therefore this offset is slightly superior to 90 degrees for a left-hand helix. This offset is also finely tuned manually for now as it can change between different microrobot as well as surface state.

The control formula for $\psi$ is:

$$
\psi=\operatorname{argument}(C)+\text { offset },
$$

The spintop motion presents the advantage of being more robust to flow than swimming. Indeed it is less impacted by the mainstream flow as it evolves close to the surface where the flow speed must match a null condition. However, this motion is in $2 \mathrm{D}$ and the RTS can be blocked by obstacles and can face the risk to be stuck permanently to the surface. The swimming motion can overcome this issues by using the helical shape of the microrobot to propel the RTS in 3D thanks to the liquid viscous drag.

As our tracking is based on the microscope image, the closed-loop control of swimming motion is limited to the horizontal plane. For now, we propose a closed-loop control on the image plane while an open-loop maintains the RTS at the same altitude by compensating its weight. Therefore, we cannot prevent a small drift of the RTS that slightly changes its altitude with time.

For non-neutral buoyant microrobots, the speed due to gravity adds to the propulsive speed. So the fluid drag which opposes the total speed, has components along both the microrobot direction and perpendicular to this direction. due to the linearity of the flow equation at low Reynold number, the drag $(D)$ can be explicit by the following equation

$$
D=-a_{\|} V_{\|}-a_{\perp} V_{\perp},
$$

with $a_{\|}$and $a_{\perp}$ the drag coefficients respectively parallel and perpendicular to the microrobot direction. $V_{\|}$and $V_{\perp}$ are the components of the microrobot"s speed parallel and perpendicular to the microrobot direction.
A closed loop control of the propulsion and direction using a component of a helical microrobot thrust for gravity compensation has already been demonstrated (Mahoney et al. 2011). For this study we are concerned by controlling the velocity in the horizontal plane. Therefore we have only developed an open loop control to compensate the gravity where the component of the thrust on the vertical axis is set to compensate the vertical falling speed measured during the characterisation of the microrobot. This open loop leads to slight drift in the vertical direction over time.

For the closed loop control, we can consider the projection of the speed on the horizontal plane (which corresponds to our microscope image plane) and by assuming the flow parallel to the RTS direction:

$$
V_{h}=p \omega \cos \theta,
$$

with $p=-b / a_{\|}, a_{\|}$and $b$ correspond here to the first two coefficients of the propulsion matrix (Purcell 1977). In our case $b$ is positive but would be negative for a right hand microrobot.

In practise $p$ is measured just before the experiment to guarantee that intrinsic properties of the RTS are the same (magnetization, shape, quantity of magnetic material). Indeed we experimentally observed that the value of $p$ could evolve with time (Barbot et al. 2017) The measurement process of $p$ is partially automatic as the user only has to drive the RTS with the same direction for few seconds while holding a certain key on the keyboard used for control. In this case the microrobot direction needs to be horizontal so the viscous drag on the horizontal plane is only affected by $a_{\|}$

Thanks to the linearity of the propulsion force with the rotation speed and our assumption on the viscous drag , we can use a simple PID controller by decoupling each direction of space $(x, y, z)$ as shown in Figure $3 \mathrm{~b})$. On each of these directions, we express the propulsion force in the corresponding rotation frequency $\left(\omega_{x}, \omega_{y}, \omega_{z}\right) . \omega_{x}$ and 
Spintop closed-loop

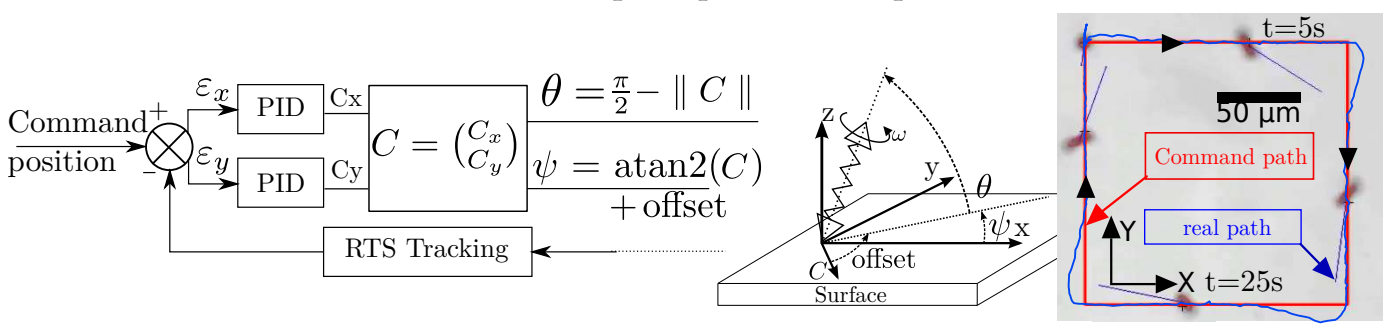

a)

Swimming closed-loop

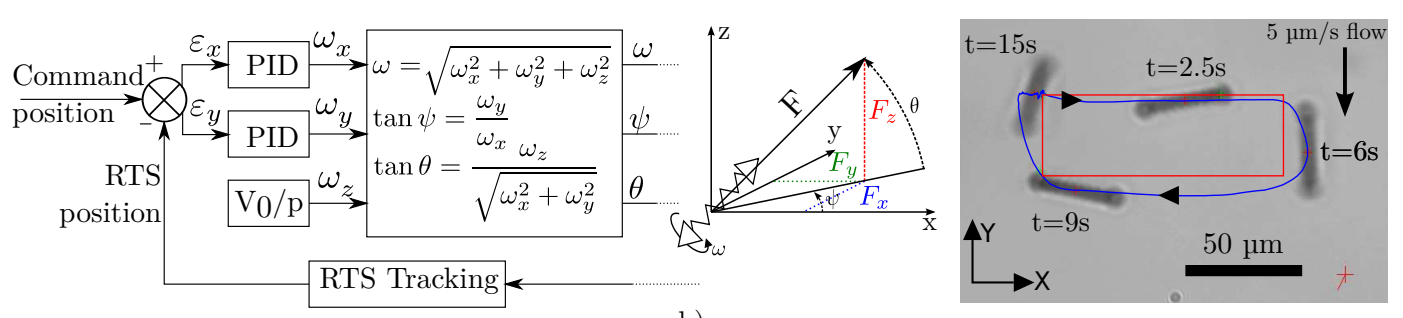

b)

Figure 3. Closed-loop control diagram and experimental top view photomontage for spintop motion and swimming motion

$\omega_{y}$ are calculated with a PID controller fed with the error on $x$ and $y$ between the tracked position and the command position. $\omega_{z}$ is the needed value to overcome gravity and it is given by:

$$
\omega_{z}=\frac{v_{0}}{p},
$$

with $v_{0}$ the falling speed of the RTS when the rotation frequency is forced to $0 \mathrm{~Hz}$. This value is experimentally determined once for each new RTS in an isolated chamber of the microfluidic chip to prevent parasite flows. Five different measurements are made to check the repeatability. The typical $0 \mathrm{~Hz}$ speed for our RTS model is around $7.5 \mu \mathrm{m} \cdot \mathrm{s}^{-1}$ . The standard deviation for a single microrobot is below 0.3 $\mu \mathrm{m} \cdot \mathrm{s}^{-1}$.(Barbot et al. 2017)

As the frequency is proportional to the force and therefore the microrobot speed, the control information on $\left(\omega_{x}, \omega_{y}, \omega_{z}\right)$ can be linearly assembled to give the control in terms of $(\omega, \psi, \theta)$ :

$$
\begin{gathered}
\omega=\sqrt{\omega_{x}^{2}+\omega_{y}^{2}+\omega_{z}^{2}}, \\
\tan \psi=\frac{\omega_{y}}{\omega_{x}}, \\
\tan \theta=\frac{\omega_{z}}{\sqrt{\omega_{x}^{2}+\omega_{y}^{2}}},
\end{gathered}
$$

with $\omega$ the rotation frequency of the magnetic field, which corresponds to the rotation frequency of the RTS as the control is made under the step-out frequency (Barbot et al. 2016), $\psi$ the yaw angle corresponding to the direction of the microrobot in the horizontal plane and $\theta$ the pitch angle corresponding to the angle between the microrobot direction and the horizontal plane.

Figure 3 b) also presents a step-by-step photomontage of the experimental microscope view during a swimming closed-loop on a rectangular path. During the experiment a flow around $5 \mu \mathrm{m} \cdot \mathrm{s}^{-1}$ was pushing the RTS along the $y$ direction. This explains the difference between the real path and the command path on the $y$ axis. More details on the command and real position evolution with time are available in Appendix B.2.

\section{Local Flow Measurement}

\section{Principle}

Figure 4 shows how we can deduce the speed of the flow in the horizontal plane from the RTS theoretical speed. For this, we use a measurement of the absolute speed obtained with the image tracking from the camera. Then we subtract from this speed the theoretical speed to obtain the drift of the RTS. This corresponds to the flow speed. The theoretical speed is calculated using the parameter $p$ which linearly links the rotation frequency to the helical microrobot theoretical speed (see equation 4). As explained in the previous section, this parameter is obtained by measuring the horizontal speed of the RTS when there is no flow.

Figure 4 also explains how this calculation is made in our control software. It is important to note that to get more precision, we directly use the value of the yaw angle from the tracking. Indeed as the amplification and electromagnetic coil stages do not have a perfectly linear behavior, small errors in the command direction can happen and induce an error in the theoretical RTS speed direction.

This flow measurement principle could be easily extended to measure vertical flow. The only limitation for this is to develop an altitude tracking. With such tracking, the theoretical vertical speed could be compared to the actual vertical speed. Note that the theoretical value should be compensated by the vertical speed due to gravity and buoyancy effects.

\section{Experimental Demonstration}

To demonstrate the flow sensing ability of the RTS, we propose to analyze two different flow profiles due to different channel geometries inside the microfluidic chip. Figure 5 a) 


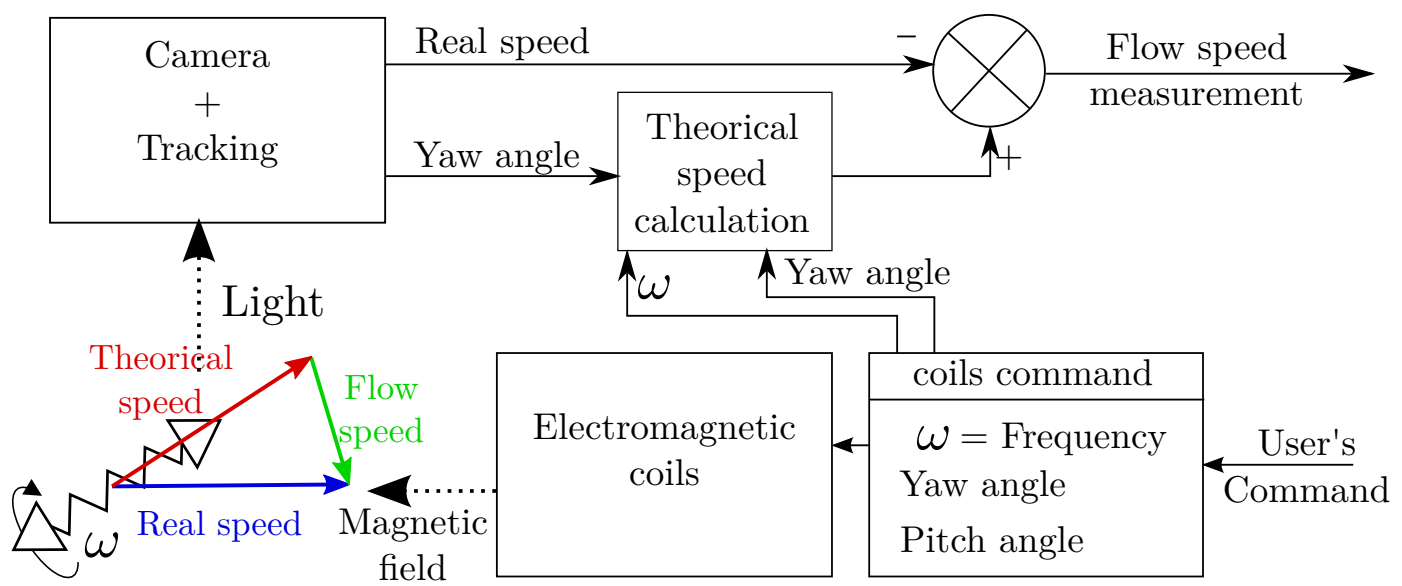

Figure 4. Online flow measurement principle and implementation. Note that the yaw angle is directly measured on the microrobot instead of using the input value. This allows us not to suffer from the error introduced by the electromagnetic setup.

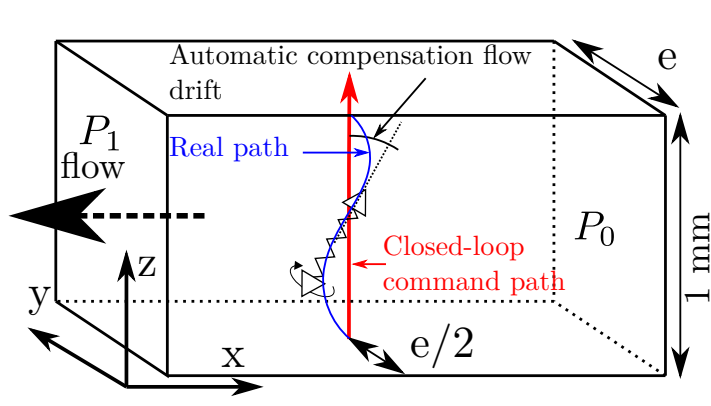

a)

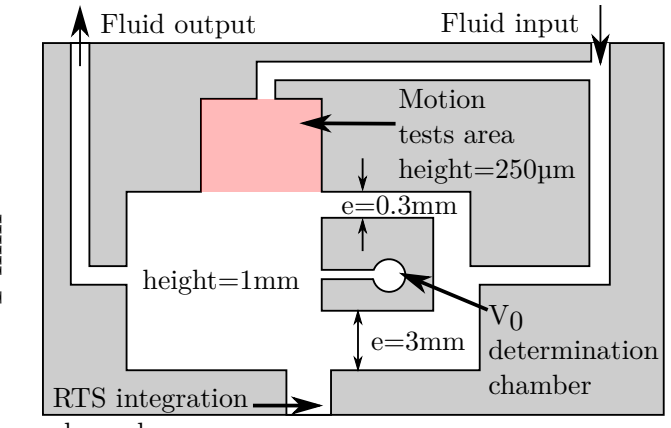

b)

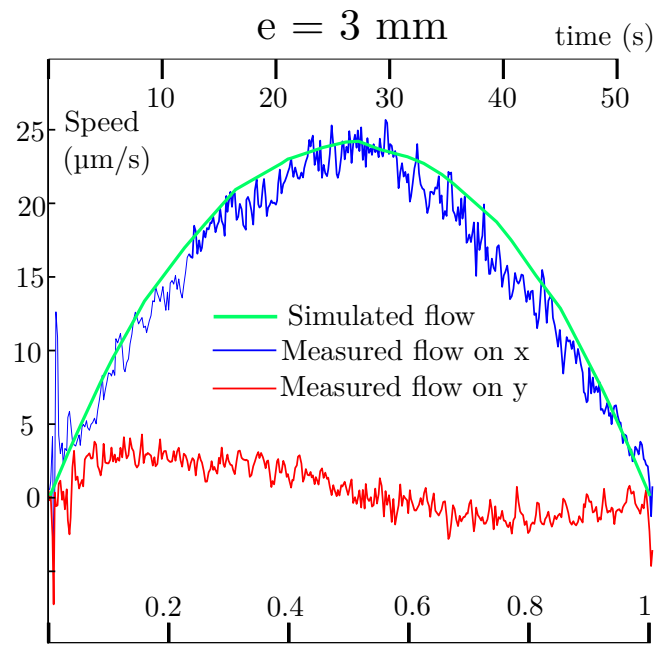

c)

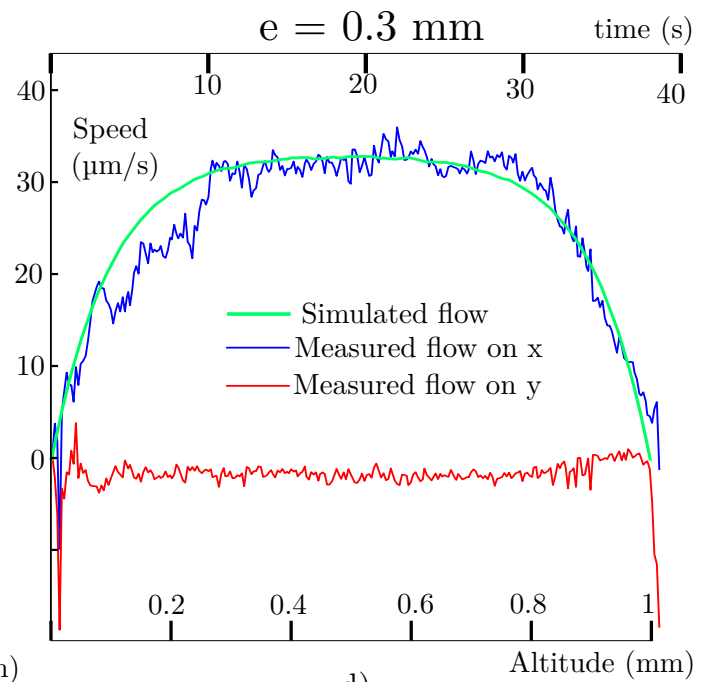

d)

Figure 5. RTS local flow measurement results. a) shows the closed-loop control path command inside channels to measure profiles, b) presents a schematic top view of the microfluidic chip. It displays the configuration to allow two channels with different widths (e), an isolated chamber for $v_{0}$ determination and area for motion adaptation tests. c) and d) display the result of the measure with a theoretical flow profile which is scaled to match the maximum flow speed

displays the geometry of these two profiles. They are both rectangular section channels with a $1 \mathrm{~mm}$ height. The width, represented by $e$ in Figure 5 a), is the only difference between the two channels. One has a width of $0.3 \mathrm{~mm}$ and the other of $3 \mathrm{~mm}$. Both sides of the channel are respectively connected to the microfluidic input and output. These connections are connected to two reservoirs with different pressures. Therefore, this generates a pressure difference between the microfluidic input and output which results in a flow inside the channel.

This geometry allow a flow speed component only in the horizontal plane. In Figure 5 a) the path of the RTS is displayed in red. This path is in the middle of the channel and goes from the bottom to the top. The closed-loop control maintains the RTS centered on this path, the vertical component of the propulsion remains the same. Therefore, 
the vertical speed of the RTS can be considered constant. In a post-process step this speed is measured precisely by determining the time for the RTS to go from the bottom to the top of the channel. During the experiment the altitude of the microfluidic chip is moved by a motorized microstage commanded manually to keep the RTS in the optical focus plane.

Figure $5 \mathrm{~b}$ ) shows the two different locations of the measurement inside the microfluidic chip to provide two square channels with different width. On this figure we also display the position of the isolated chamber used to make the experiment to determine the falling speed of the RTS $\left(v_{0}\right)$. This speed is an essential parameter of the altitude openloop swimming control. We also mention the position of the area where the tests to adapt the motion depending on the flow condition are performed. Supplemental Video 2 shows a experimental view record of one flow profile measurement.

Figures 5 c) and d) show the flow profiles measurement for the two different channel widths $e$. As expected, a parabola shape is obtained for $e=3 \mathrm{~mm}$. Indeed in this case the lateral walls are too far to have any influence on the flow and the solution is close to the 2D one with infinite $e$ value. For $e=0.3 \mathrm{~mm}$, the walls have a large influence and the solution is closer to a square shape flow. To provide a comparison, we simulate with a finite element software (Comsol multiphysics) the expected flow profile for the two channels. The Stokes equation was used to compute the flow by taking the IPA (Isopropyl alcohol) viscosity at $20^{\circ} \mathrm{C}$. The obtained profiles are scaled to match the maximum value of the experimentally measured profiles. Therefore, as the flow is linear, the flow profile is the same for any pressure shift between $P_{0}$ and $P_{1}$. We take it equal to 1 bar. We see that the measured shapes fit well the simulated ones.

The position errors of the swimming closed-loop control was below $15 \mu \mathrm{m}$ for $\mathrm{x}$ positions. This error is mainly caused by the difficulties of the PID controller to keep the RTS at the same position as the flow intensity changes with the altitude. This error does not impact the measurements as the flow profile is invariant with $\mathrm{x}$. For $\mathrm{y}$, the error is below $2 \mu \mathrm{m}$ and we can therefore consider its impact to be negligible. The error evolution with time is displayed in Appendix B.

The difference between the experiments and the simulations as well as the relatively strong noise can be mainly attributed to the tracking algorithm. As presented in the previous section on the swimming closed-loop control, the change in RTS direction impacts the tracking position. Therefore, an error is made on the RTS speed when its direction changes. Moreover if the focal plane is not perfectly set in the middle of the RTS, either the top or the bottom will be more in focus thus resulting in the displacement of the tracking center. This error can also be important as we manually track the optical plane which creates another artificial speed of the RTS added directly to the measurement.

The error is also due to the drift approximation which consider the flow parallel to the microrobot direction. Indeed the microrobot have a small speed component due to the gravity. Therefore, the error on the measurement is below $10 \%$. The detail of the error development in this case is presented in the Appendix B.3. For now this error is less important than the instability of the tracking, but in case this method should be used for precise flow measurement a precise characterisation of the microrobot to determine its perpendicular and parallel drag coefficients should be realised in order to correct this error.

Finally some error could also arise when the RTS is near a wall as surfaces impact the flow distribution and thus the propelling force. However, this error decays quickly far from the surface and is generally already below $10 \%$ after 2 body length $\mu \mathrm{m}$ (Spagnolie and Lauga 2012) which is sufficient to detect on overflow scenario. In the RTS case, we proved in a previous work that the impact of the wall of the speed along the wall direction is below $3 \%$ for a $20 \mu \mathrm{m}$ distance (Barbot et al. 2017) (supplementary material G). So the error from the surface impact is for now negligible compared to the tracking noise, which should be the first step to improve for better measurement. Then more complex measurement paths could be developed to measure the flow at a specific location with the RTS going into different directions. By doing so the loss or gain of propulsion due to the surface could be extracted as well as a corrected flow value. The presented results of this paper seem satisfactory enough for a proof of concept. We believe they are enough to guarantee that we can use this method to measure the local flow at the RTS position in order to automatically detect dangerous overflow situation.

\section{Local Flow Measurement for Semi-Automatic Motion Adaptation}

\section{Motion Choice Algorithm}

To limit the risk of the RTS to be carried away by the flow and to improve its robustness, we want to develop a semiautomatic control. The user could benefit from their high level understanding of the physics and the situation in order to define the best path in the chip. Then the RTS control system could compute the safest motion along this path. Therefore, we propose here to develop such algorithm to ensure the safest RTS displacement along a predefined path.

To design this algorithm, we first need to see how dangerous situations presented in Figure 1 can be avoided. Unfortunately the surface stiction is for now difficult to detect in advance. We hope that further research on the friction between the RTS and the surface based on the Appendix B.1 results will propose a solution to anticipate the probability of the RTS to be stuck to the surface. However, an excessive flow can easily be detected. Therefore, the best motion decision algorithm can be summarized for now in the following sentence: "If swimming is possible then use swimming."

Figure 6 explains how this algorithm is implemented in our current system. In swimming motion the RTS follows the path by closed-loop control. If the flow measurement exceeds a given threshold the state changes and the RTS switches to "swimming to spintop motion" state. In this state the RTS aims toward the ground at the fastest speed. This flow threshold depends only on the microrobot speed and can be chosen by the maximal acceptable drift angle between the propulsion and the direction. Therefore, the threshold is given by the following formula :

$$
\text { Threshold }=\frac{\text { speed }}{\tan (\text { drift })}
$$




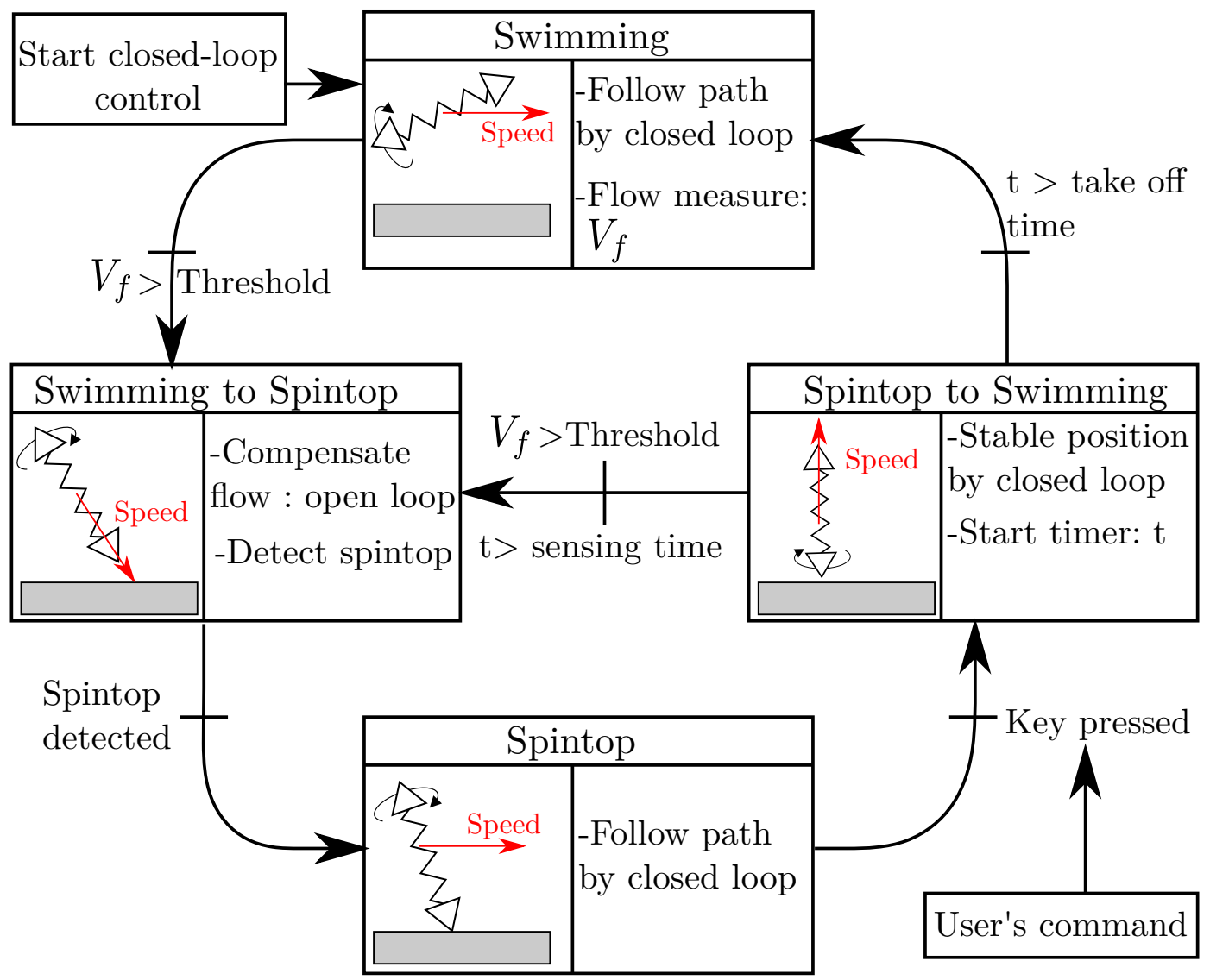

Figure 6. Algorithm to guarantee the safest motion along a path.

It is safer to pick an angle below 45 degrees (i.e a threshold speed below the microrobot speed) as some destination becomes unreachable when the maximal drift exceed 45 degrees. Then the collision with the ground is detected by evaluating the difference between the axis of the RTS and its direction projected on the horizontal plane. In swimming this difference is close to zero whereas it is close to $\pi / 2$ in spintop motion and correspond to the offset presented in Figure 3 a). The last measured flow speed is used to determine a direction that will partially compensate the flow drift.

When spintop motion is detected the closed-loop control on the path is restored and the RTS continues to follow the path in spintop motion. The decision of trying to return to swimming is for now made by the user. Indeed they are in the best position to determine if the flow speed has reduced. However, to reduce even more the time spent in spintop motion and therefore the probability of getting stuck to the surface, triggering periodically the command to try to return in swimming is probably the optimal strategy. In general cases, the frequency of the take off attempt should be at least the double of the maximum flow change frequency in order to not miss any swimming opportunity.

In ideal cases, the swimming altitude should be the lowest as possible to be less impacted by the flow as well as reducing the necessary time to switch to a friction based motion. A minimum safe distance to avoid surface contact is one body length of the microrobot. However, there is a need to add to this distance a margin corresponding to the maximum error of the altitude control to take into account of the altitude control error as well as the maximum roughness of the surface. As we do not have yet an altitude tracking, we evaluate this altitude with the time assuming a constant vertical speed. Therefore a minimum value for the take off time can be given by the following formula:

$$
\text { take off time }>\frac{L \sin \theta+m+\epsilon}{v},
$$

with $\mathrm{L}$ the body length of the microrobot, $\theta$ the maximum pitch angle in the trajectory as defined in Figure 4, $m$ the maximum roughness of the surface, $\epsilon$ the tracking error and $v$ the set vertical speed.

In our experiment the consecutive swimming time of the RTS was restrained to 40 seconds and the maximum error of the gravity compensation was $1 \mu \mathrm{m} \cdot \mathrm{s}^{-1}$ This leads to $\epsilon=40 \mu \mathrm{m}$. Moreover $L=50 \mu \mathrm{m}$ and $m=0$, to be sure to cover every configuration of the microrobot we also consider $\theta=\pi / 2$. Therefore according to equation 10 , a minimum take off time of 4.5 seconds was required by setting a vertical speed of $20 \mu \mathrm{m} \cdot \mathrm{s}^{-1}$.

In practice we choose to wait for 5 seconds for the RTS to reach a $100 \mu \mathrm{m}$ altitude for swimming. At the end of this time the RTS comes back to the swimming state and continues to follow the path by closed-loop control with the swimming motion.

A constant horizontal position during the "spintop motion to swimming" state is maintained by the swimming closed-loop algorithm. The RTS compensates the flow and maintains the same $x$ and $y$ position during the "spintop motion to swimming" state. As the RTS is in swimming 


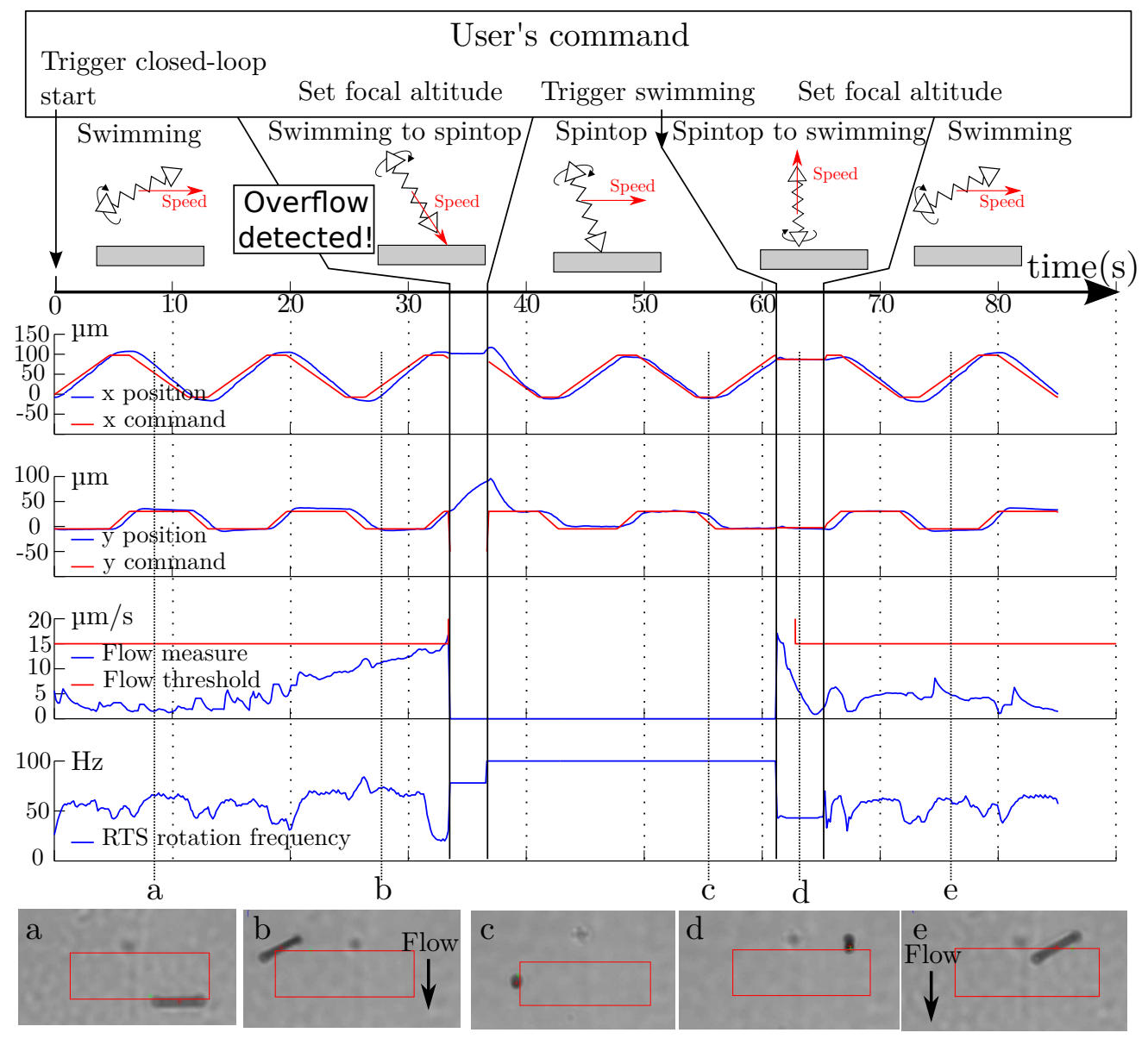

Figure 7. Semi-automatic motion adaptation along a cyclic rectangular path. A record of this experiment is presented in the trial 2 of Supplemental video 3. The flow speed increases until the flow threshold is reached and the RTS switches to spintop motion motion. Then the flow is reduced and the RTS is manually triggered to change to swimming motion. For several time markers, an experimental view is displayed: a) swimming with no flow, b) swimming by compensating the flow, c) spintop motion, d) transition from swimming to spintop motion, e) swimming by compensating the flow.

mode during the ascension, the flow can be measured. If it becomes larger than the flow threshold, the RTS returns to the ground by switching to the "swimming to spintop motion" state. Therefore, trying to switch to swimming is guaranteed without risk of losing the RTS on the flow and can be tried as often as one needs.

The flow measurement is perturbed by the surface proximity when the RTS takes off. Therefore, there is some delay in the algorithm before starting the flow measurement after take off. To improve robustness, this delay needs to be as small as possible, especially if strong flow is present. Assuming that we have a spheroidal microrobot with the propulsion distributed on most of the surface, the perturbation become lower than $25 \%$ at half a body length of the surface (Spagnolie and Lauga 2012). Therefore a good indication for starting the sensing time before the measurement can be given by the following expression :

$$
\text { sensing time } \approx \frac{L}{2 v}
$$

In our case this give a waiting time of 1.25 seconds, that we decide to round to 1 second. Indeed, even if some error can still be made on this measurement, it is better to have a rough flow estimation quickly in case the flow value is clearly larger than the threshold.

\section{Experimental Demonstration}

To test this algorithm we propose to command the RTS on a repeated rectangular path. This repetition of path is required as we have not implemented a moving platform to follow the RTS on the chip on a long closed-loop control path. This gives us time to tune the flow in order to test the motion adaptation algorithm. To precisely tune this flow, the input reservoir is put on a platform where the altitude can be set manually with millimeter precision to vary the pressure. As both input and output reservoir surfaces are connected to the ambient pressure, the elevation of the input reservoir creates a pressure difference that generates the flow. To reduce the noise of the flow measurement by the RTS, we perform an averaging of its value on one second.

Figure 7 shows the results for this path. In this figure, experimental views are presented at different times. As we do not have an altitude tracking system, the focus is set manually. At first the RTS follows the path thanks to the swimming closed-loop control as it can be seen in Figure 7 picture a). The altitude is around $100 \mu \mathrm{m}$. At the end of the first rectangular path we start to increase the flow gradually. At the beginning the RTS can continue to perform the swimming motion and the PID controller automatically compensates the drift due to the flow. The picture b) is taken 
at this time. We see that the RTS is not parallel to the path to compensate the flow drift. Then the flow value reaches the threshold which is set to $15 \mu \mathrm{m} \cdot \mathrm{s}^{-1}$. This triggers the transition to spintop motion and the RTS control switches to the state: "swimming to spintop motion". The closed-loop control is stopped and the RTS aims for the ground. Even if the direction of the RTS is set to compensate the flow, some errors in the position appear in the $y$ position.

When the RTS touches the ground, the closed-loop control is restored in spintop motion. As the propulsion is controlled by the pitch angle in this motion, the frequency is set to $100 \mathrm{~Hz}$. The flow measurement becomes impossible and is therefore stopped. The picture c) is taken during this spintop motion closed-loop control. To test the ability of the RTS to return to swimming, we reduce the flow. Then we manually trigger the transition to the "spintop motion to swimming" state which restores the swimming closedloop control. The picture d) is taken during this state. The horizontal position is kept constant as the vertical speed is set to $20 \mu \mathrm{m} \cdot \mathrm{s}^{-1}$ to reach an altitude around 100 $\mu \mathrm{m}$. After one second in this state, the flow threshold is reactivated to detect potential overflow. As it can be seen on the flow measurement curve, the take-off from spintop motion to swimming creates perturbations that increase the flow estimation artificially. This justifies waiting one second before restoring the flow threshold. After five seconds the desired altitude of approximately $100 \mu \mathrm{m}$ is reached, because we set the ascending speed to $20 \mu \mathrm{m} \cdot \mathrm{s}^{-1}$ in the altitude open-loop. Then the closed-loop controller on $x$ and $y$ is set to follow the path and the altitude open-loop on a $0 \mu \mathrm{m} \cdot \mathrm{s}^{-1}$ speed. As it can be seen in Figure 7 e) the swimming state is restored. Supplementary Video 3 shows the record corresponding to these results.

In some cases where we would like to restore the swimming motion, the flow speed is still over the threshold and too dangerous for swimming motion. Therefore, it is important to prevent the RTS from reaching the swimming state and to be carried away by the flow. Figure 8 a) shows a record corresponding to this situation. Here the RTS first follows the rectangular path with the closed-loop control of the spintop motion. Then the user triggers the transition to swimming and the RTS control switches to the "spintop motion to swimming" state. After one second the flow threshold is set and then the measurement flow reaches this threshold. This triggers the transition to the "swimming to spintop motion" state until the RTS returns in contact with the ground and continues to follow the path by closed-loop controlled spintop motion. The video of this experiment is available in Supplementary Video 4.

Finally, Figure 8 b) shows what happens if the RTS control does not perform motion adaptation. In this experiment the RTS follows the path by swimming motion and the flow is continuously increased. At first, the RTS control can compensate the flow drift but as the necessary frequency gets higher than $200 \mathrm{~Hz}$, the RTS reaches its step out rotation frequency and the speed no longer increases with the frequency. This happen for a flow speed around $30 \mu \mathrm{m}$. $s^{-1}$. The RTS speed decreases brutally after the step-out. This results in an almost immediate loss of the microrobot from the frame and a definite potential loss. The rapidity of this phenomenon demonstrates the advantage of automatic motion control as a human user cannot monitor at all times the motion of the RTS to conclude in a too large flow situation. The video of this experiment is available in Supplementary Video 5.

It can be seen on all the experimental records that the flow measurement presents strong fluctuations and noise. These errors are due to perturbations in tracking during the brutal change of direction of the RTS while following the rectangular path. Therefore, to reduce it, the solution would be to improve the tracking precision.

In laminar regime, the flow intensity reduced near the surface, therefore one good strategy to avoid strong flow is to move close to the surface. This can be especially interesting for helical microrobots with an almost neutral buoyancy and therefore a small maximum pitch to overcome gravity. For such case, surface impact on the microrobot drag and therefore the flow measurement could be impacted. So in this case an experimental tuning of the microrobot would be necessary to compensate this measurement error.

\section{Conclusion}

In this paper we first proposed automatic control of the swimming motion improving flow robustness. This automatic control relies on two closed-loop controls for the $x$ and $y$ coordinates and one open-loop control based on the RTS characterization for the $z$ coordinates. This open-loop control was necessary as we currently have no way to track the RTS altitude online. Then this automatic closed-loop control was used to measure the flow profile in rectangular channels with different widths. These flow profiles obtained by the RTS were shown to match the shapes obtained by numerical simulations. Finally, we showed that a semiautomatic algorithm with minimum user intervention could switch from swimming to spintop motion (which is a surface motion less impacted by the flow in order to keep a path control in various flow conditions). As no information on the flow can be obtained in spintop motion, the signal to trigger the return to swimming motion remains, for now, a manual input from the user.

Indeed the ability to detect unsafe surfaces where the microrobot could easily become stuck to the surface would also improve the general robustness in a full automatic control. Improvement on the image analysis could provide obstacle detection in order to swim over them. For high obstacles, changing the focus of the lens could be used to determine their altitude whereas obstacles smaller than the depth of focus of the field could be detected by contrast change in the image.

Finally, to continue improving the robustness of the RTS, the motion algorithm could also include the rolling motion. In this motion the RTS is in contact with the surface along all of its length and therefore it is even less dependent on the flow. However, this motion is more likely to result in the microrobot becoming permanently stuck on the surface of the microfluidic channel.

We think this work is the first step to allow a simple user control interface for helical microrobots where no prior knowledge of the microrobot control and particularities is required. The user input should be limited only by the desired position of the RTS. Ultimately, the system should 


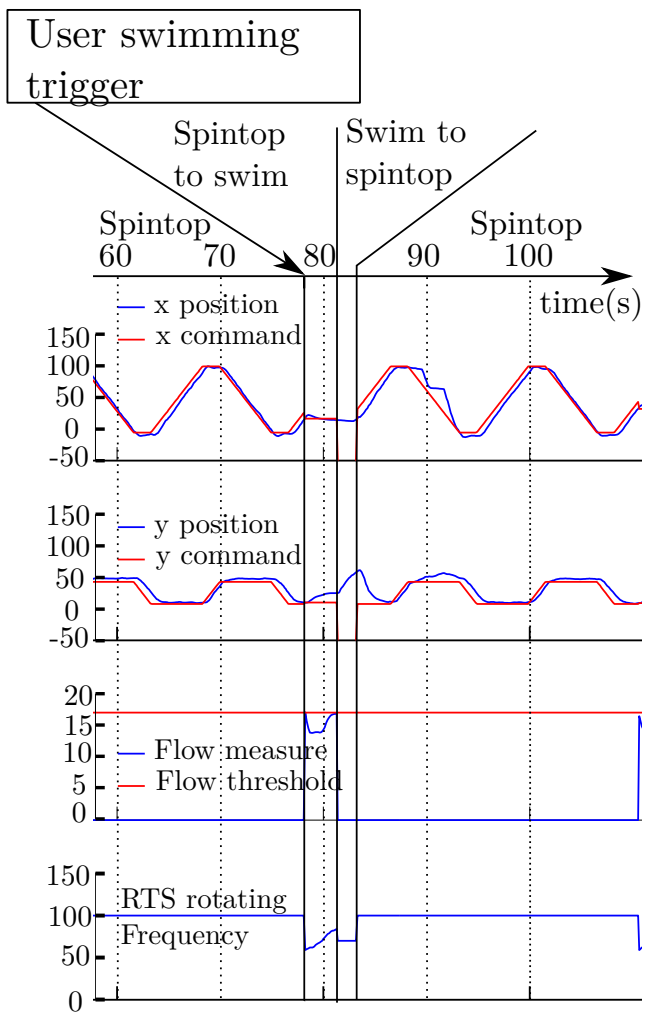

a)

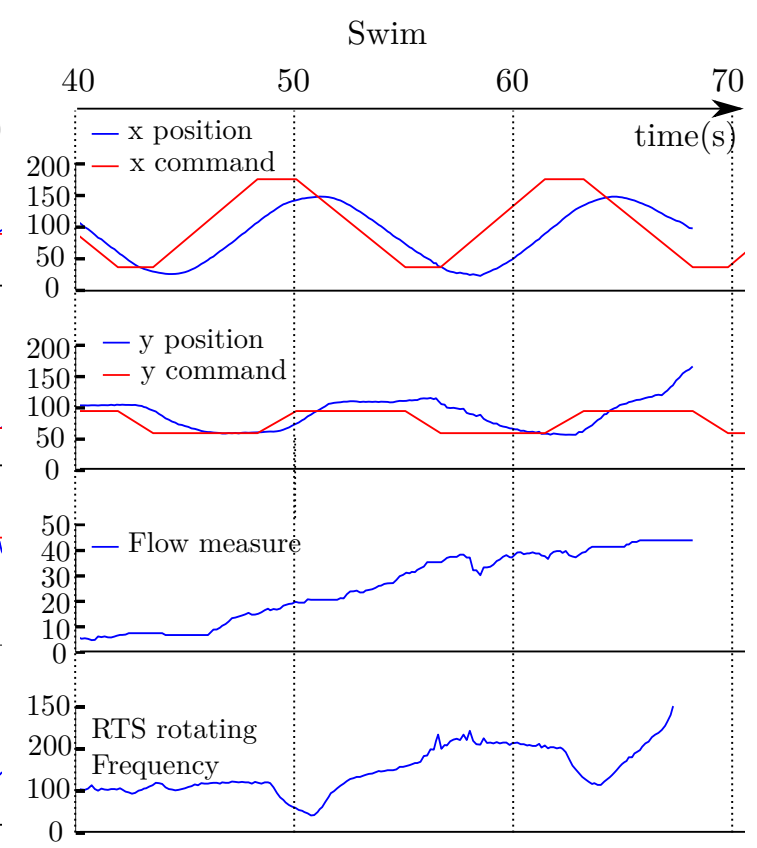

b)

Figure 8. a) shows the RTS in spintop motion with a flow superior to the threshold. When the transition to swimming is triggered the algorithm quickly detects the overflow and comes back to spintop motion. The video of this experiment is available in supplemental video 4. b) shows the RTS swimming without motion adaptation. As the flow increases the RTS control saturates and the RTS is carried away by the flow. The video of this experiment is available in Supplementary Video 5.

choose the best motion to safely go to this location. If this location is too dangerous to be reached safely by any means, the motion should be stopped and a warning displayed to the user.

\section{References}

Barbot A, Decanini D and Hwang G (2016) On-chip Microfluidic Multimodal Swimmer toward 3D Navigation. Scientific reports 6(January): 19041. DOI:10.1038/srep19041. URL http: //www . nature.com/srep/2016/160121/ srep19041/full/srep19041.html.

Barbot A, Decanini D and Hwang G (2017) Helical microrobot for force sensing inside microfluidic chip. Sensors and Actuators A: Physical 266: 258-272.

Hagiwara M, Kawahara T, Yamanishi Y and Arai F (2011) Precise Control of Magnetically Driven Microtools for Enucleation of Oocytes in a Microfluidic Chip. Advanced Robotics 25(8): 991-1005. DOI:10.1163/016918611X568611. URL http: //www.tandfonline.com/doi/abs/10. 1163/016918611X568611.

Honda T, Arai KI and Ishiyama K (1996) Micro swimming mechanisms propelled by external magnetic fields. IEEE Transactions on Magnetics 32(5 PART 2): 5085-5087. DOI: 10.1109/20.539498.
Kawahara T, Sugita M, Hagiwara M, Arai F, Kawano H, ShihiraIshikawa I and Miyawaki A (2013) On-chip microrobot for investigating the response of aquatic microorganisms to mechanical stimulation. Lab on a chip 13(6): 1070-8. DOI:10. 1039/c2lc41190c. URL http://www.ncbi.nlm.nih. gov/pubmed/23314607.

Leong TG, Randall CL, Benson BR, Bassik N, Stern GM and Gracias DH (2009) Tetherless thermobiochemically actuated microgrippers. Proceedings of the National Academy of Sciences of the United States of America 106(3): 703-708. DOI:10.1073/pnas.0807698106.

Li J, Sattayasamitsathit S, Dong R, Gao W, Tam R, Feng X, Ai S and Wang J (2013) Template electrosynthesis of tailored-made helical nanoswimmers. Nanoscale 6(April 2016): 9415-9420. DOI:10.1039/c3nr04760a. URL http: / /www . ncbi . nlm. nih.gov/pubmed/24126904.

Mahoney AW, Sarrazin JC, Bamberg E and Abbott JJ (2011) Velocity Control with Gravity Compensation for Magnetic Helical Microswimmers. Advanced Robotics 25(8): 1007-1028. DOI:10.1163/016918611X568620. URL http://www.tandfonline.com/doi/abs/10. 1163/016918611X568620.

Medina-Sánchez M, Schwarz L, Meyer AK, Hebenstreit F and Schmidt OG (2016) Cellular Cargo Delivery: Toward Assisted Fertilization by Sperm-Carrying Micromotors. Nano Letters 16(1): 555-561. DOI:10.1021/acs.nanolett.5b04221. 
Mhanna R, Qiu F, Zhang L, Ding Y, Sugihara K, Zenobi-Wong M and Nelson BJ (2014) Artificial bacterial flagella for remotecontrolled targeted single-cell drug delivery. Small 10(10): 1953-1957. DOI:10.1002/smll.201303538. URL http:// www.ncbi.nlm.nih.gov/pubmed/24616145.

Purcell E (1977) Life at low Reynolds number. Am. J. Phys 45(3): 11. DOI:10.1119/1.10903. URL http://www . hep.princeton.edu/ $\{\sim$ mcdonald/examples/ fluids/purcell\{_\}ajp\{_\}45\{_\}3\{_\}77.pdf.

Qiu F, Fujita S, Mhanna R, Zhang L, Simona BR and Nelson BJ (2015) Magnetic Helical Microswimmers Functionalized with Lipoplexes for Targeted Gene Delivery. Advanced Functional Materials 25(11): 1666-1671. DOI:10.1002/adfm.201403891.

Spagnolie SE and Lauga E (2012) Hydrodynamics of selfpropulsion near a boundary: predictions and accuracy of farfield approximations. Journal of Fluid Mechanics 700: 105147.

Srivastava SK, Medina-Sánchez M, Koch B and Schmidt OG (2016) Medibots: dual-action biogenic microdaggers for single-cell surgery and drug release. Advanced Materials 28(5): 832-837.

Tottori S, Zhang L, Qiu F, Krawczyk KK, Franco-Obregõn A and Nelson BJ (2012) Magnetic helical micromachines: Fabrication, controlled swimming, and cargo transport. Advanced Materials 24(6): 811-816. DOI:10.1002/adma. 201103818. URL http://www.ncbi.nlm.nih.gov/ pubmed/22213276.

Tung HW, Sargent DF and Nelson BJ (2014) Protein crystal harvesting using the RodBot: A wireless mobile microrobot. Journal of Applied Crystallography 47(2): 692-700. DOI: 10.1107/S1600576714004403.

Xi W, Solovev AA, Ananth AN, Gracias DH, Sanchez S and Schmidt OG (2013) Rolled-up magnetic microdrillers: towards remotely controlled minimally invasive surgery. Nanoscale 5(4): 1294-1297.

Ye Z, Diller E and Sitti M (2012) Micro-manipulation using rotational fluid flows induced by remote magnetic micromanipulators. Journal of Applied Physics 112(6). DOI: 10.1063/1.4754521.

Zhang L, Petit T, Lu Y, Kratochvil BE, Peyer KE, Pei R, Lou $\mathrm{J}$ and Nelson BJ (2010a) Controlled propulsion and cargo transport of rotating nickel nanowires near a patterned solid surface. ACS Nano 4(10): 6228-6234. DOI:10.1021/ nn101861n. URL http://www.ncbi.nlm.nih.gov/ pubmed/20873764.

Zhang L, Peyer KE and Nelson BJ (2010b) Artificial bacterial flagella for micromanipulation. Lab on a chip 10(17): 22032215. DOI:10.1039/c004450b. URL http://www. ncbi. nlm.nih.gov/pubmed/20567752.

\section{Appendix A: Index to multimedia extension}

Table of multimedia extension

\begin{tabular}{lll}
\hline Extension & Media type & Description \\
\hline 1 & video & Swimming closed-loop control \\
\hline 2 & video & $\begin{array}{l}\text { Channel flow profile } \\
\text { measurement }\end{array}$ \\
\hline 3 & video & $\begin{array}{l}\text { Control algorithm demonstration: } \\
\text { Adaptation to overflow }\end{array}$ \\
\hline 5 & video & $\begin{array}{l}\text { Control algorithm demonstration: } \\
\text { Spintop motion to swimming } \\
\text { transition aborded by strong flow }\end{array}$ \\
\hline \multirow{2}{*}{ video } & $\begin{array}{l}\text { Loss of the swimming RTS in an } \\
\text { only swimming closed-loop } \\
\text { control scheme }\end{array}$
\end{tabular}

\section{Appendix B}

\section{B.1 Spintop motion different regimes}

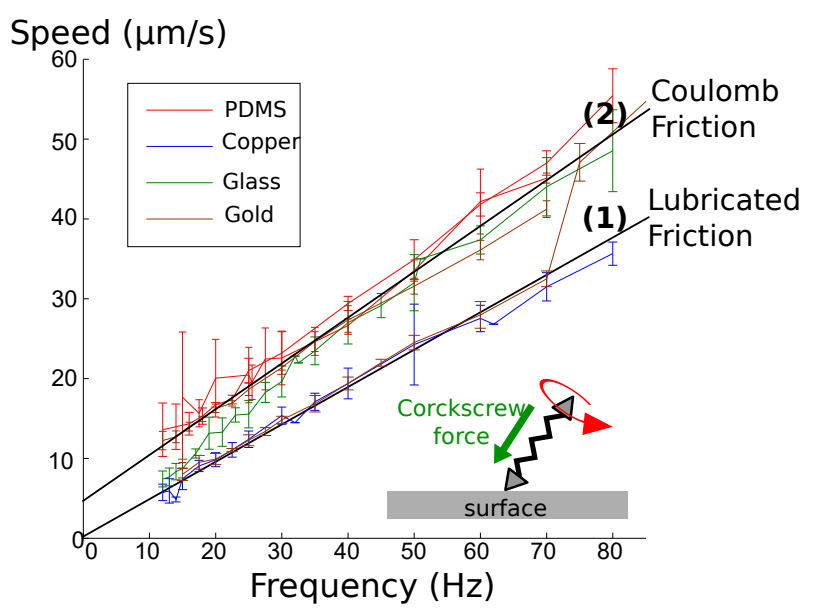

a)

Figure 9. The two different spintop motion regimes on different substrate. The speed following the tendency 1 ) is well explained by a lubricated friction model whereas the tendency 2) can be explained by a coulomb surface model.

Figure 9 shows the evolution of the RTS speed with the frequency where the force provided by the helical part is pushing the RTS into the substrate. To get these results, we integrate several substrates coated with different materials into the main chamber of the microfluidic chip. The metallic substrates are in glass with a $100 \mu \mathrm{m}$ thickness and the coating is made with a PVD process with a thickness of $10 \mathrm{~nm}$. To integrate these substrates into the chip we first bond on the glass cover, a thin sheet of PDMS $(\approx$ $100 \mu \mathrm{m}$ ) with a total surface around $25 \mathrm{~mm}^{2}$. Then we cut the different substrates in $2 \mathrm{~mm}^{2}$ square parts and bond them to the PDMS sheet. Finally, the microfluidic chip is bonded to the glass and the main chamber is placed to contain the PDMS sheet and the different substrates. As this main chamber thickness is $1 \mathrm{~mm}$ different substrates are still accessible to the RTS by swimming.

Then the inlet and outlet of the chip are closed to limit parasite flows and the RTS uses swimming motion to reach the different substrates and perform spintop motion. Unfortunately during the experiment a small parasite flow around $5 \mu \mathrm{m}$ was still present. To analyze these data we consider that the friction point between the RTS 
and the surface is providing all the propelling force that counterbalances the fluid drag exerted by the fluid. This fluid drag is considered as proportional to the speed thanks to the low Reynolds number approximation.

From the curves in Figure 9, we see that two different linear tendencies seem to emerge. They are referred as (1) and (2) and their linear approximations are drawn. For the gold surface, two similar experiments were performed but different results were obtained. One follows the tendency (2) and the other follows the tendency (1) until $70 \mathrm{~Hz}$ where it brutally changes for the tendency (2). The reasons of the RTS to be in one of the two regimes is still an open question.

Even if it is not possible for now to understand the reasons of the RTS to switch between these two tendencies and how much this depends on the surface composition, we can still make some interesting assumptions on the underlying physics. The main difference between these two linear tendencies is that when the frequency is null, (1) seems to extrapolate to a null value and (2) to a value around $5 \mu \mathrm{m} \cdot \mathrm{s}^{-1}$. This difference can be explained by different friction models. The tendency (1) seems to be well explained by a lubricated model where liquid remains between the RTS and the surface. In this case, the friction on one object is obtained only by the fluid action on this object. The friction force is then given for a low Reynolds flow by the following equation:

$$
d F=\nu \cdot \frac{\partial u}{\partial n}
$$

where $d F$ is the force element at a given point, $\nu$ the viscosity, $u$ the speed of the fluid and $n$ the normal axis to the surface on the point.

As we are in a low Reynolds flow, the flow speed at the boundary must match the speed of the objects. In this case, if the rotating speed of the RTS is null, the flow speed will also be null at every point and so will be any of its space derivatives. The low Reynolds number approximation also guarantees linearity which means that the rotation speed of the RTS is linearly linked to the flow speed and thus to the RTS speed. Hence the linearity and the null value at $0 \mathrm{~Hz}$ seem well explained by this lubricated model. The presence of a liquid layer between the RTS and the surface seems therefore a convincing hypothesis for the tendency (1).

The tendency (2) seems to fit a Coulomb kinematic friction scheme. This well-established empirical model proposes an interpretation for the increase in surface contact due to the normal force between two objects. It is given by the following equation:

$$
F=K \cdot F_{N},
$$

where $F$ is the friction force, $K$ the kinematic friction coefficient between the two materials and $F_{N}$ the force normal to the contact between the two objects. This model explains also the affine trend of the curves (2). Indeed the RTS rotation frequency is linearly linked to the normal force thanks to the helical shape that maintains a pushing force on the substrate. However, the main justification of this model is that it explains the positive value of the affine approximation of the curve at zero frequency. Indeed the force between the RTS and the surface is the sum of the corkscrew force and the weight of the RTS. Therefore, by extrapolating this model to $0 \mathrm{~Hz}$, some speed should remain, corresponding to the friction force only due to the weight of the RTS. Thus, we can conclude that the friction is in part governed by Coulomb model. In fact in the case (2) we cannot suppress the influence of the propulsion coming from the viscosity which explains the tendency (1). Indeed there is no reason for this viscous force to completely disappear. Therefore, the lateral force resulting in the speed of the tendency (2) is probably a mix of the viscous drag and solid friction modeled by the Coulomb law.

The switch between these two regimes during one of the gold surface experiments as well as the gradual change from one regime to another in the gold experiment in Figure 9 gives credit to these contact model hypotheses. Indeed in both cases the transition happens from the lubricated model to the physical contact model with the increase of frequency, i.e. with the increase in the force that pushes the RTS on the substrate. It seems logical that increasing this force tends to suppress the liquid layer between the two objects.

\section{B.2 Swimming Closed-loop Control Time Response}

Figure 10 displays the evolution of the command and real position with time during swimming closed-loop control . We see that the RTS converges to the command position when it stops moving. However a relatively large error and delay are present for a moving command. Unfortunately we saw experimentally that a more reactive system presents a risk of being unstable and therefore the PID controller could not be tuned to reduce more this error.

\section{B.3 Flow Channel Measurement: Position Errors}

The Figure 11 displays the distance between the RTS and the control point during the flow measurement experiment. 


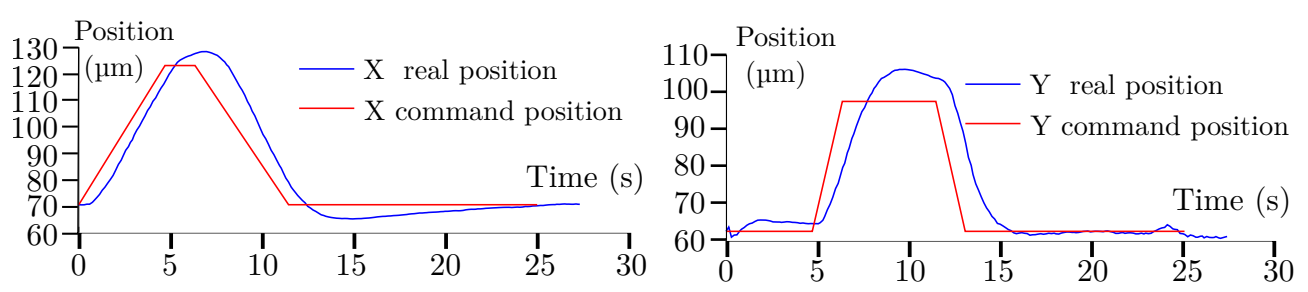

Figure 10. Command and real position of the RTS control in closed-loop while swimming.

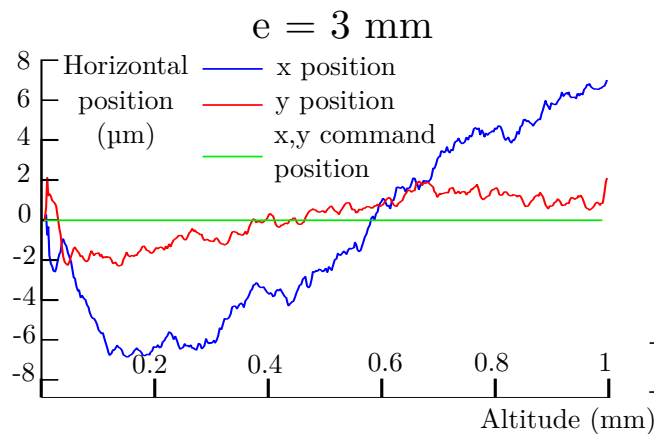

a)

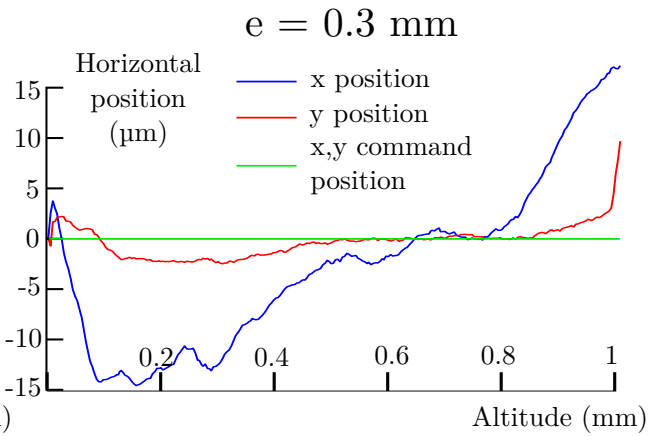

b)

Figure 11. $x$ and $y$ positions errors during channel flow profile measurement, $x$ is in the direction of the flow. 